\title{
PART 8.
}

\section{X-Ray Binaries, Transients And Super-Soft Sources}




\title{
Kilohertz QPO in Low Mass X-Ray Binaries: an Overview
}

\author{
M. van der Klis \\ Astronomical Institute "Anton Pannekoek" and Center for High-Energy \\ Astrophysics, University of Amsterdam, Kruislaan 403, $1098 \mathrm{SJ}$ \\ Amsterdam, The Netherlands
}

\begin{abstract}
I review the current status of the investigations of the kilohertz quasi-periodic oscillations that have been discovered in low-mass Xray binaries (LMXBs) with NASA's Rossi X-ray Timing Explorer (RXTE) since February 1996. Seven sources have now shown this new phenomenon, one $\mathrm{Z}$ source (Sco X-1) and 6 atoll sources (all X-ray bursters), and some patterns are beginning to emerge. The frequencies of the oscillations are between 500 and $1200 \mathrm{~Hz}$. They can be coherent for more than $10^{2}$ cycles, but a more typical coherence is several $10^{1}$. Amplitudes are between 0.5 and $16 \%$ (rms) of the total flux. Most sources show double $\mathrm{kHz}$ peaks separated by a few $10^{2} \mathrm{~Hz}$, which move up and down in frequency together. In Sco X-1 the peak frequencies systematically increase, and the peak separation systematically decreases, with $\dot{M}$. In $4 U 1728-34$ a third oscillation is seen at a frequency equal to the difference frequency of the two $\mathrm{kHz}$ peaks, but only during $\mathrm{X}$-ray bursts. A simple interpretation in terms of a beat-frequency model with the difference frequency being the neutron star's spin, as suggested by this result, is inconsistent with the fact that in Sco X-1 the peak separation varies.
\end{abstract}

\section{Introduction}

The recent discovery of kilohertz quasi-periodic oscillations (QPO) in low-mass X-ray binaries (LMXB) with the Rossi X-ray Timing Explorer (RXTE) constitutes the first reliably detected, persistent (although not always present), phenomenon in accreting stellar-mass compact objects whose time scale approaches the dynamical time scale of the compact object. The highest frequency so far reported is $1170 \mathrm{~Hz}$ (see Table 1); if this would be the frequency of a Keplerian orbit around a $1.4 M_{\odot}$ neutron star (which we don't know), this orbit would have a radius of $\sim 15 \mathrm{~km}$, which is smaller than the star's radius predicted by the softest equations of state. The $\mathrm{kHz}$ QPO are also one of the fastest macroscopic phenomena observed outside the solar system.

\section{Current status of the observations}

Figures 1 and 2 show two examples of $\mathrm{kHz}$ QPO peaks in power spectra of Xray data obtained with RXTE. Two important characteristics are illustrated by these figures: the fact that sometimes the peaks are quite narrow, indicating 


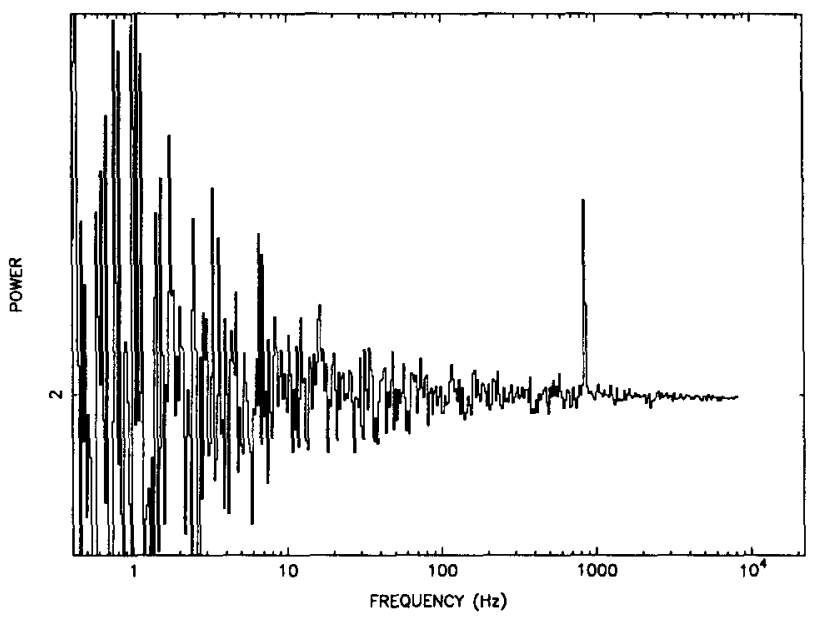

Figure 1. A power spectrum of $4 \mathrm{U} 1608-52$ showing a thin QPO peak near $860 \mathrm{~Hz}$. The $\mathrm{kHz}$ QPO peak in this source can be as narrow as $4 \mathrm{~Hz}$, with amplitudes of up to $20 \%$ (rms) at photon energies above $12 \mathrm{keV}$ (Berger et al. 1996, see Table).

coherence times of more than $10^{2}$ cycles, and the fact that often there are two peaks.

Table 1 summarizes the observations of the $\mathrm{kHz}$ QPO peaks that have been reported up to now. The lower and upper peaks are tabulated separately; in cases where two peaks were seen simultaneously, they appear on one line in the table; in cases where only one peak was seen in the power spectrum, the identification as lower or upper was performed on the basis of correlations with total source intensity. Sometimes no decision was possible as to whether a peak was a lower or an upper peak; these frequencies are tabulated in between the lower and upper frequency columns. Reported peak separations have, where possible, also been listed, as have oscillations in X-ray bursts. Actually observed frequency variations (as opposed to differences in frequency in different observations) are indicated with arrows.

Peak widths are in the range $<0.01-\sim 0.1$ times the centroid frequency; the amplitudes can be as large as $2.5 \%$ (rms) of the total flux in Sco X-1 and $16 \%$ in some of the atoll sources. In most sources, the peak frequencies systematically increase with $\dot{M}$; among the sources that have shown frequency variations, there is no strong evidence for this only in $4 \mathrm{U} 1608-52$. In $4 \mathrm{U} 1728-34,4 \mathrm{U} 1636-53$ and $4 \mathrm{U} 1608-52$ the QPO amplitude increases from a few percent near $3 \mathrm{keV}$ to $10-15 \%$ above $12 \mathrm{keV}$; in $4 \mathrm{U} 0614+091$ the QPO spectrum appears to be much less hard. In Sco X-1, and perhaps also 4 U 1728-34 the peak separation decreases with $\dot{M}$; in $4 \mathrm{U} 1636-53$ it may increase. In $4 \mathrm{U} 1728-34$ and perhaps $4 \mathrm{U} 0614+091$ nearly coherent oscillations are seen in bursts at a frequency close to the peak separation frequency. 


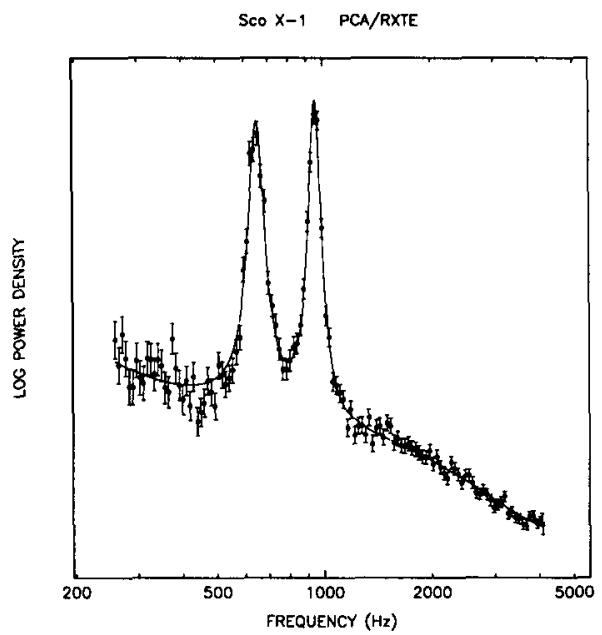

Figure 2. A power spectrum of ScoX-1 showing the double-peaked structure that characterizes much of the $\mathrm{kHz}$ QPO data. The sloping continuum is dominated by instrumental deadtime effects. The $\mathrm{kHz}$ QPO peaks in Sco X-1 have been observed to move by $\sim 20 \%$ in frequency. When the frequency increases, the peak separation decreases (van der Klis et al. 1996, see Table).

\section{Models}

Various models are being considered for this new phenomenon. The three frequencies in $4 \mathrm{U} 1728-34$, one being the difference of the two others, strongly suggest a beat-frequency interpretation (Strohmayer et al. 1996, see Table), where $\nu_{\text {beat }}=\nu_{\text {Kepler }}-\nu_{\text {spin }}$, with the lower QPO peak at $\nu_{\text {beat }}$, the upper one at $\nu_{\text {Kepler }}$ and the burst oscillation at $\nu_{\text {spin }}$. However, in its simple form where the observed $\nu_{K e p l e r}$ is the same as the one that beats with the spin to produce the beat, this model requires the peak separation to be constant, which is definitely not true for Sco X-1. We can not exclude yet that we have different $\mathrm{kHz}$ QPO phenomena, but in view of the many coincidences in the phenomenology this idea seems unattractive.

A model where the $\mathrm{kHz}$ QPO are a Doppler-shifted version of a millisecond X-ray pulsar signal (van der Klis et al. 1996, see Table) might work. If the Doppler-shifting takes place in two symmetric relativistic jets of speed $v$, then the QPO frequencies are $\nu_{\mp}=\nu_{\text {pulse }}(1-v / c) /(1 \pm(v / c) \cos \theta)$, where $\theta$ is the angle between the jets and the line of sight. The ScoX-1 data fit $\nu_{\text {pulse }} \sim 1370 \mathrm{~Hz}$ (this could be twice the spin frequency) and $\theta \sim 65^{\circ}$, with $v$ varying between 0.28 and $0.46 c$. Of course precession could produce variations in $\theta$. Doppler boosting and disk obscuration of the receding jet can explain why the lower peak is often weaker than the upper one, and sometimes absent. This model has no obvious explanation for the third frequency in $4 \mathrm{U} 1728-34$. 
Table 1. Observed frequencies of kilohertz QPO.

\begin{tabular}{|c|c|c|c|c|c|}
\hline $\begin{array}{l}\text { Source } \\
\text { (in order } \\
\text { of RA) }\end{array}$ & $\begin{array}{c}\text { Lower } \\
\text { peak } \\
\text { freq. } \\
(\mathrm{Hz}) \\
\end{array}$ & $\begin{array}{c}\text { Upper } \\
\text { peak } \\
\text { freq. } \\
(\mathrm{Hz}) \\
\end{array}$ & $\begin{array}{c}\text { Peak } \\
\text { sepa- } \\
\text { ration } \\
(\mathrm{Hz})\end{array}$ & $\begin{array}{l}\text { Burst } \\
\text { oscill. } \\
\text { freq. } \\
(\mathrm{Hz})\end{array}$ & Ref. \\
\hline $4 U 0614+091$ & $\begin{array}{c}500 \\
\downarrow \\
600\end{array}$ & $\begin{array}{c}580 \\
630 \\
730 \\
820 \\
\downarrow \\
930\end{array}$ & $327 \pm 4$ & $326^{a}$ & $\begin{array}{l}1 \\
2 \\
2 \\
1 \\
1 \\
1\end{array}$ \\
\hline $4 U 1608-52$ & \multicolumn{2}{|c|}{$\begin{array}{c}830 \\
\downarrow \\
890\end{array}$} & & & $\begin{array}{l}3 \\
3 \\
3\end{array}$ \\
\hline$S \operatorname{co} X-1$ & $\begin{array}{c}570 \\
\downarrow \\
800 \\
\downarrow \\
830\end{array}$ & $\begin{array}{c}870 \\
\downarrow \\
1050 \\
\downarrow \\
1080 \\
\downarrow \\
1130\end{array}$ & $\begin{array}{c}292 \pm 2 \\
\downarrow \\
247 \pm 3\end{array}$ & & $\begin{array}{l}4 \\
4 \\
4 \\
4 \\
4 \\
4 \\
4\end{array}$ \\
\hline $4 U 1636-53$ & \multicolumn{2}{|c|}{$\begin{array}{c}835 \\
\downarrow \\
885\end{array}$} & $\begin{array}{c}225 \pm 20 \\
\downarrow \\
290 \pm 5\end{array}$ & & $\begin{array}{l}5 \\
5 \\
5 \\
2 \\
2 \\
2\end{array}$ \\
\hline $4 U 1728-34$ & $\begin{array}{c}640 \\
\downarrow \\
715 \\
\downarrow \\
790\end{array}$ & $\begin{array}{c}500 \\
\downarrow \\
990 \\
\downarrow \\
1060 \\
\downarrow \\
1100\end{array}$ & $\begin{array}{c}351 \pm 7 \\
\downarrow \\
342 \pm 12\end{array}$ & 363 & $\begin{array}{l}6 \\
6 \\
6 \\
6 \\
6 \\
6 \\
6\end{array}$ \\
\hline KS 1731-260 & & & & 524 & 7 \\
\hline $4 U 1735-44$ & & 50 & & & 8 \\
\hline
\end{tabular}

References. 1: Ford et al. 1996 IAUC6426, Kaaret et al. 1996 these proc.; 2: Van der Klis et al. 1996 IAUC6428, Mendez et al. 1996 in prep., Wijnands et al. in prep.; 3: Van Paradijs et al. 1996 IAUC 6336, Berger et al. 1996 ApJ 469 L13; 4: Van der Klis et al. 1996 IAUC 6319,6424, ApJ 469 L1, and in prep.; 5: W. Zhang et al. 1996 ApJ 469 L17; 6: Strohmayer et al. 1996 IAUC 6320,6387, ApJ 469 L9; 7: Morgan et al. 1996 IAUC 6437; 8: Wijnands et al. 1996 IAUC 6447 and in prep.

a "Marginal." 